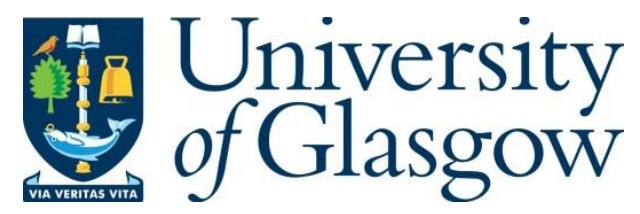

Mathur, N., Glesk, I., Davidson, A., Paul, G., Banford, J., Irvine, J. and Buis, A. (2016) Wearable Mobile Sensor and Communication Platform for the In-situ Monitoring of Lower Limb Health in Amputees. In: 2016 IEEE International Symposium on Circuits and Systems (ISCAS), Montréal, QC, Canada, 22-25 May 2016, pp. 1366-1369. ISBN 9781479953417.

There may be differences between this version and the published version. You are advised to consult the publisher's version if you wish to cite from it.

http://eprints.gla.ac.uk/197288/

Deposited on: 5 March 2020

Enlighten - Research publications by members of the University of Glasgow http://eprints.gla.ac.uk 


\title{
Wearable Mobile Sensor and Communication Platform for the In-situ Monitoring of Lower Limb Health in Amputees
}

\author{
Neha Mathur*, Ivan Glesk*, Alan Davidson*, Greig Paul*, Jamie Banford**, James Irvine*, Arjan Buis ${ }^{+}$ \\ *Department of Electronic and Electrical Engineering, University of Strathclyde, Glasgow G1 1XW, UK \\ ${ }^{+}$Department of Biomedical Engineering, University of Strathclyde, Glasgow G4 0NW, UK \\ ** formerly of the Department of Electronic and Electrical Engineering, University of Strathclyde, Glasgow G1 1XW, UK
}

\begin{abstract}
Monitoring the health of a residual limb in prosthesis is key to detect early signs of tissue injury, and could improve patient quality of life. However, monitoring at skin level could induce possible discomfort and irritation, making the skin susceptible for breakdown. The use of non-invasive sensor technologies within the flexible liner of the prosthetic device can alleviate these issues. The rehabilitation of lower limb prosthesis wearers can be greatly improved by a reliable continuous monitoring system that can alert both the user and health authority by early warning of the development of tissue damage. In this work, we have created a wearable sensor platform for lower limb amputees that is capable of gathering data from the sensors (placed on the elastomer), and store and transmit to a central health database, for the purpose of analyzing it. This paper describes the wearable mobile platform for data collection from flexible sensors embedded in prosthetic liner and describes the system architecture used, as well as some of the challenges related to the collection of 'big data'.
\end{abstract}

Keywords - e-health; elastomer; lower limb prosthetics; rehabilition; sensors; tissue health; wearable sensor platform

\section{INTRODUCTION}

The incidence of limb amputation is increasing worldwide, particularly in wealthier western nations. Limb amputations result not only from trauma, but also increasingly from the complications of disease. In particular, trends toward poor diet and lifestyle choices and an ageing population in the western nations have led to an increase in many diseases, including type-2 diabetes and ischaemic heart disease. In particular, poorly managed diabetes can lead to devastating medical complications such as infection, gangrene, Charcot foot disease and poor circulation, leading to the amputation of digits or limbs. Indeed, diabetes has become the single biggest cause of amputation in United Kingdom, with about 7000 foot amputations per year [1]. It would therefore be of great benefit to prosthetic users in general and, in particular lower limb diabetics; to be able to detect either the early signs of actual tissue injury before the development of serious complications; and/or monitor the conditions at the prosthetic socket/residual limb interface to give a warning of a significant increase in the risk of injury before it develops. A reliable continuous monitoring and early warning system that can alert both the user and health authority would reduce admissions to hospital, reduce the associated costs, improve patient quality of life and perhaps allow a significant reduction in the frequency of outpatient check-up appointments. In addition, the information provided by a monitoring system on areas prone to damage could contribute toward improving prosthesis design. Such a monitoring system is envisaged to consist of sensor arrays distributed, for example, within a prosthetic socket or footwear either as an integral part of the socket/footwear or as or shoe. While the technologies and principles considered as candidates are not new, to date no such early warning system has been developed and, as such a continuous monitoring system to provide an early warning of tissue injury presents a novel approach to injury prevention. However, many issues arise in the design and implementation of such a monitoring system, not least of which is the continuing ability of ICT (information and communication technology) networks to provide the quality of service required to ensure that patient monitoring data is reliably conveyed to the relevant health authority.

e-health has grown rapidly in recent years [2]. In case of critical m-health (mobile health) monitoring systems, it is vital that patient data is not lost and in some cases that patient data reach the relevant authority with minimal delay to avoid life threatening complications. This involves scenarios where the patient may require to be monitored continuously, or emergency alerts may be critical to patient welfare, such as in case of deep tissue injury formation. However, issues with mobile communications channel availability and issues with latency and limited bandwidth in data networks and data network equipment may threaten the transmission of this data to the health authority.

In our work we identify non-invasive sensor technologies that may be suitable for use within the prosthetic socket environment to measure the bio-markers/quantities determined above, taking additional account of the necessary constraints imposed by sensor system size/weight and sensor/skin contact considerations. Also, the architecture and design of our wearable sensor platform is capable of monitoring, logging and transmitting the patient data to a central health authority, is discussed. The data-sets recorded using these systems are then processed using machine learning algorithms to extract clinically relevant information from the data. The capability of

This work was supported by the Engineering and Physical Sciences Research Council (EPSRC) under the Doctoral Training Grant and the Center for Excellence in Rehabilitation Research (CERR). Also, support for the climate chamber was given by University of Glasgow. 
the wearable sensor platform is also tested on an amputee subject and the results are presented in the subsequent sections.

\section{SENSOR TECHNOLOGIES}

Obtaining physiological data like temperature, pressure, oxygenation levels and gait by placing the sensor directly in contact with the skin would give the most accurate readings, but this would lead to practicality issues in the prosthesis, such as protruding wiring and consistent positioning of sensors. Also it could induce possible skin irritation and discomfort. On the other hand, non-invasive measurement by embedding the sensors in the elastomer or hard socket material of the prosthetic device could be a solution to unobtrusively gather data from multiple sensors. The sensors can be then interfaced with the wearable platform.

The major challenge of embedding the sensors within the liner of the prosthetic device is to maintain high performance while keeping the system flexible. These systems need to respond like conventional electronics and at the same time confirm to complex three-dimensional structures. Integrating the desired sensors and electronics within the flexible silicone membrane (prosthetic liner, PDMS) is an achievable but challenging task in terms of ensuring flexibility, while at the same time avoiding mechanical deformation [3]. If not done properly, the embedding process into the flexible material of the prosthesis can result in elastomer failures due to the high strain induced when donning a liner. It should also be noted that these flexible silicone liner materials may influence the response of the embedded sensors and such influence must be decoupled by smart signal processing to retrieve the desired data.

The principles of piezoelectricity are widely applied as the basis for pressure and force measurement transducers. Piezoelectric materials are a class of dielectrics that have asymmetric crystalline structures and that can be polarized by an electric external electric field or by the application of a mechanical pressure. Thus, the charge produced is proportional to the pressure applied. In some materials such as PVDF (polyvinylidene fluoride) and P(VDF-TrFE) (Poly(vinylidene fluoride trifluoroethylene)), no piezoelectric effect exists in the material before poling. Both PVDF and P(VDF-TrFE), have been successfully used as pressure sensors for several years [4], and more recently in energy harnessing. Piezoelectric devices require no power supply, since a charge is developed across the piezoelectric material proportional to the applied force, thus producing an output voltage which may be measured directly. However, a drawback in their application for use within prosthetic sockets is that sensors used in the measurement of such useful quantities as pressure generally involve rigid semiconductors. These rigid semiconductors cannot be allowed to have prolonged contact with the skin as it may crack when stressed. It is therefore desirable to have a flexible and wearable system to overcome these difficulties. Thin film polymer pressure sensors show promise in the production of cheap and very flexible sensor arrays which are bio-compatible i.e. flexible, non-chemically reactive and resistant to corrosion [5]. The sensors, if placed strategically in the array, can help in extracting maximum information [6].

The use of the piezo-electric effect of a P(VDF-TrFE) co- polymer pressure sensor within footwear or prosthetic sockets is in developmental stage. By fabricating on, for example, a PEN (Polyethylene Naphthalate) substrate, P(VDF-TrFE) sensors are not only non-reactive but can also be made flexible, making them potentially suitable for use within a prosthetic socket or in footwear. An additional advantage of polymer piezoelectric devices is that fabrication is a low temperature process and is consequently relatively cheap. Ultimately the aim is to develop a P(VDF-TrFE) sensor array where each sensor is fabricated on the gate of a polymer field effect transistor, with PEN substrate to provide local amplification. Local amplification simplifies the charge amplification stage required for piezoelectric transducer outputs and therefore will reduce the power consumption of a wearable system.

\section{ARCHITECTURUAL OVERVIEW OF SENSOR PlATfORM}

\section{A. Hardware Overview}

The wearable platform is capable of interfacing with a number of sensors including P(VDF-TrFE) [7] currently being embedded in a prosthetic liner. However, for initial deployment in prosthetic devices, temperature and gait measurement sensors were implemented. The temperature and orientation of the residual limb of an amputee subject can be monitored by a medical team defined sampling rate. The data can be accessed on a server. For backup of the data, there is also a provision for the data to be locally stored on the SD card in the wearable sensor platform, as well as on the smartphone. Fig. 1 illustrates the schematic of the wearable platform.

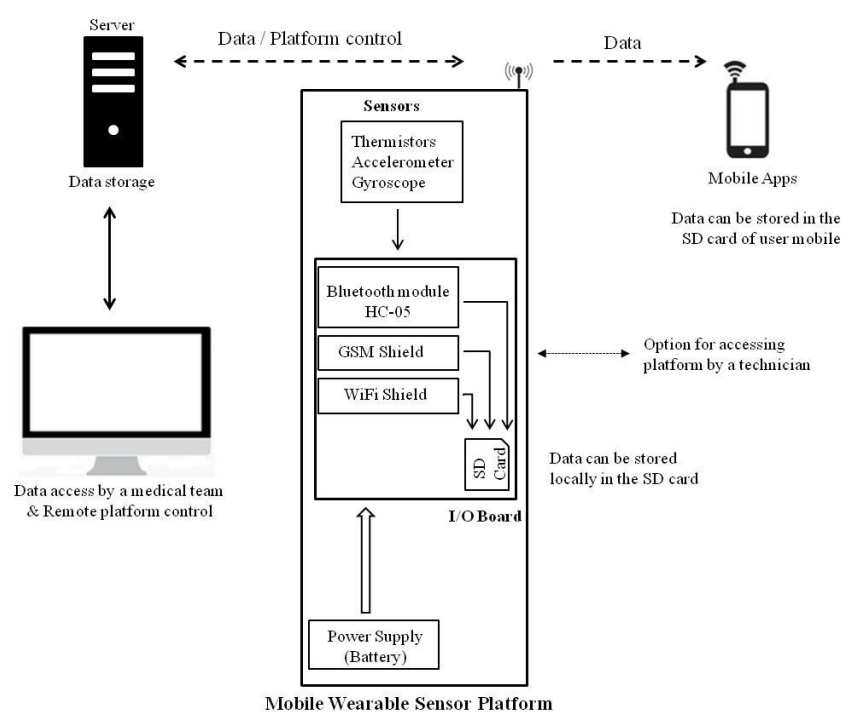

Fig. 1: Architecture of the wearable sensor platform. Adapted from [8]

Our sensing platform is composed of a number of discrete components. The core of it is a Atmel (ATmega328 16MHz) microcontroller. An MPU-6050 accelerometer and gyroscope module, along with thermistors, were used to gather movement and temperature data respectively from the residual limb, and mounted in a small housing, which could be easily attached to the amputee subject. The MPU-6050 was connected using a physical $\mathrm{I}^{2} \mathrm{C}$ connection to the microcontroller, using software 
developed on the Arduino platform. The Arduino board can communicate via Bluetooth, WiFi or cellular networks. Since the Bluetooth module has a footprint as small as $12.7 \mathrm{~mm} \mathrm{x}$ $27 \mathrm{~mm}$ with an operating voltage of $1.8 \mathrm{~V}$, it was initially selected for data communication in our wearable platform design. The microcontroller was connected to an HC-05 Bluetooth module, which communicated over Serial Port Profile (SPP). An Android smartphone was paired with this module, and connected over Bluetooth, allowing a connection to be established to our software running on the smartphone. The smartphone would then transmit the data to the monitoring server, where it was stored in a Postgres database. This was used as the back-end to a data analysis platform, allowing for retrieval and processing of the data obtained from the sensor. Fig. 2 illustrates the route of the data communication from the sensors to the central database.

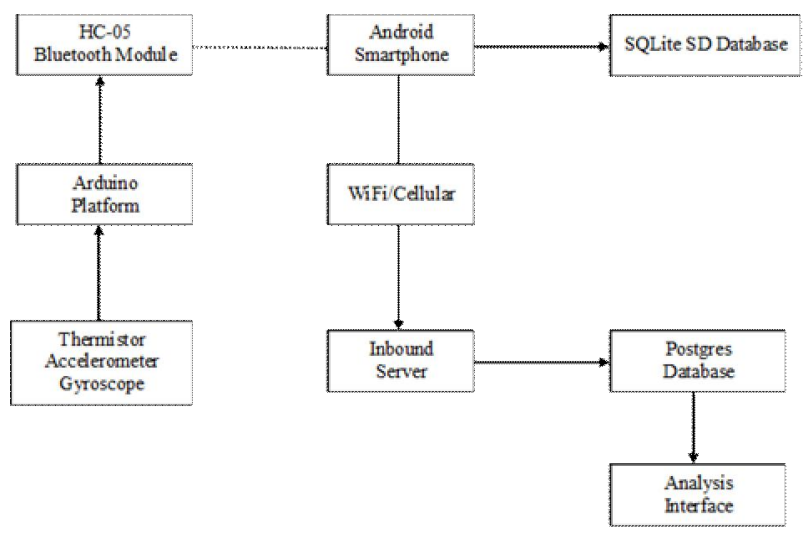

Fig. 2: Data flow in the wearable sensor platform

\section{B. Software Overview}

The Bluetooth link between the HC-05 and Android smartphone was used to transmit comma- separated readings from the sensors interfaced with it; namely thermistors, accelerometer and gyroscope. A single line of text data was transmitted for each sample. In the event of the Bluetooth connection being lost, the HC-05 buffered unsent messages, provided there was sufficient memory available, and would retransmit them upon re-establishing a connection, in order to minimize the risk of data loss due to momentary disconnection.

Within the Android application, we stored the incoming data received over Bluetooth, after tagging each sample as part of a "stream". Streams were used to identify a particular experiment being carried out, and to separate the data from each different experiment, such that it may be later analyzed.

In order to handle the scenario where experiments take place out of the range of WiFi or cellular connectivity, the Android application creates a local database storing all samples, and carries out a synchronization with the server when the connectivity is available. The synchronization logic of this process is to identify the last received sample ID for a given stream, and identify if any further samples exist for that stream (with a larger sample ID). This requires that the sample ID be monotonic and always increment over time, which was implemented in the application. To manage the load on the network interface of the device, and avoid overwhelming the server, samples were batched together in groups of 5 or 10 , and a limited number of groups of samples were sent in a single attempt.

\section{IMPLEMENTATION OF THE WEARABLE SENSOR PLATFORM}

The capability of the designed mobile wearable sensor platform was tested on a trans-tibial traumatic amputee subject in a climate controlled chamber. This investigation was carried out under ethical approval granted by the University of Strathclyde Ethics Committee (Ref UEC13/04). The subject was asked to perform a 35 minute protocol which consisted of resting (sitting) for 10 minutes, walk at a self-selected speed of 0.54 meters/second on a treadmill for 10 minutes, and finally rest for 15 minutes. The subject wore a $6 \mathrm{~mm}$ Pelite liner with a resin laminate socket with the wearable sensor platform attached on to it. Fig. 3 indicates the positioning of the wearable sensor platform in relation to the prosthesis of the subject during different activity levels. Four thermistors and an MPU-6050 module interfaced with the wearable platform, providing the residual limb and liner interface temperature profile at the lateral and medial sides and the orientation of the limb respectively. Data from the sensors was sampled at $50 \mathrm{~Hz}$ for the entire 35 minute protocol. The temperature profile of the residual limb and the liner were analysed for different ambient temperatures and it was noted that in all the cases the trace of the liner temperature was very closely correlated with that of the residual limb temperature. This enabled us to implement the Gaussian processes modelling technique to predict the residual limb temperature by monitoring the liner interface temperature with an accuracy of 95\% [9], thereby providing a non-invasive measurement practice.

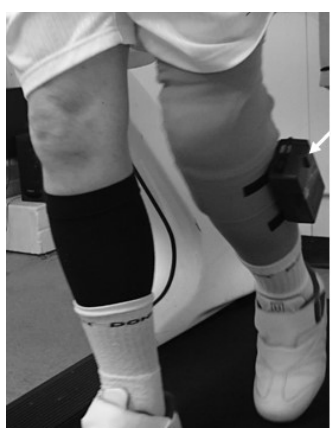

(a)

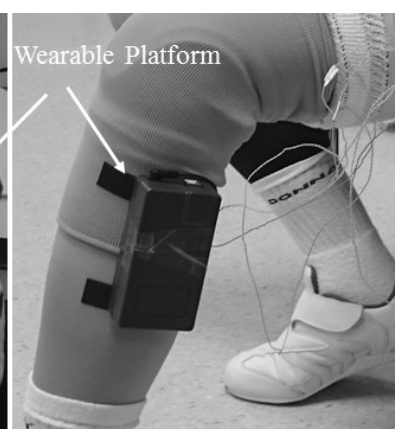

(b)
Fig. 3: The wearable sensor platform positioned on the prosthesis of the amputee subject during various activity levels as (a) walking on treadmill at a self-selected speed (b) sitting/resting

\section{DISCUSSION}

The sensor data can be accessed from a central database which is username and password protected. The data is organized by amputee subject and stream and can be downloaded for further processing. Figs. 4 and 5 indicate the acceleration and temperature profiles of the residual limb at an ambient temperature of $15^{\circ} \mathrm{C}$ which was downloaded from the web server. The data was then down-sampled in order to smoothen out the short-term fluctuations and highlight the longer-term 
trends. The data sets were then processed using machine learning algorithms and ready for use by medical professionals.

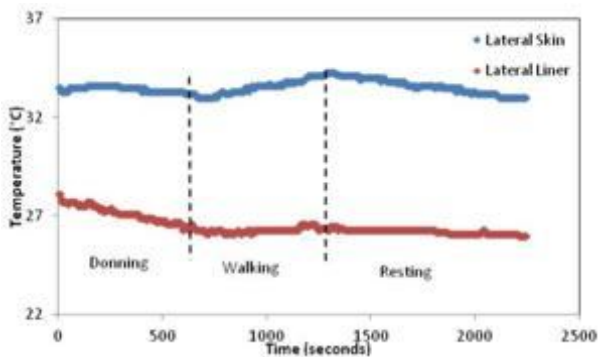

(a)

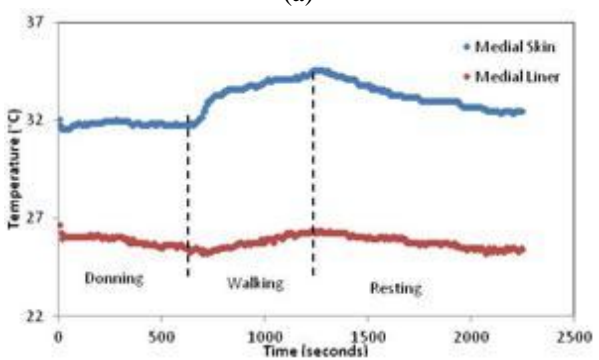

(b)

Fig. 4: Profiles of residual limb skin and liner temperatures at sampling frequency of $0.5 \mathrm{~Hz}$ with a 5 second time average on (a) lateral side (b) medial side
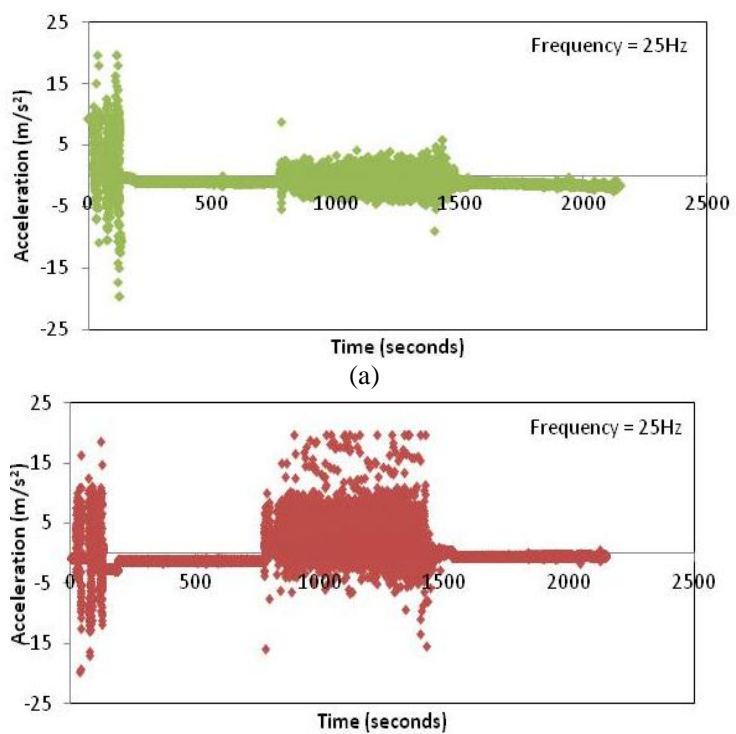

(b)

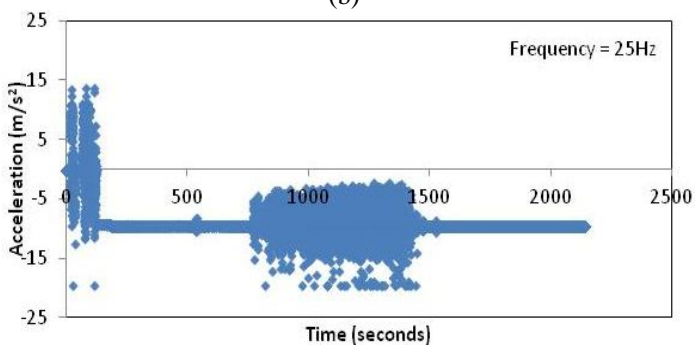

(c)

Fig. 5: Acceleration of the amputee subject while performing the experimental protocol in (a) $\mathrm{x}$-direction (b) y-direction (c) z-direction

\section{CONCLUSION}

In this paper we described a wearable mobile platform for data collection from flexible sensors embedded in a prosthetic liner. A wearable sensor communication platform has been demonstrated which is capable of harvesting data from multiple sensors for in-situ monitoring of residual limb health in amputees. This has been tested on an amputee subject (in a clinical environment) for monitoring, recording, and transmitting the temperature and gait data to a central database which can accessed securely for analysis.

In order to further reduce the power consumption and cost of the sensor unit, we have identified a number of improvements to this platform which may be made. Firstly, the power consumption of the sensor may be reduced by the use of Bluetooth Low Energy (BLE) for transmission. It would also be possible to reduce the cost and power usage by eliminating the use of the main microcontroller, if a microcontroller with integrated BLE connectivity were used. This would reduce the complexity of the design, and reduce the footprint required for the sensor unit to be installed on the prosthetic limb.

Another improvement for the performance of the solution would be a more efficient protocol for the delta-coding of samples from the sensors - since the samples were typically taken at very short intervals, the difference between adjacent sample values was small, often approaching zero, when there was limited movement. By delta-encoding these periods of little movement, the quantity of data to be transmitted could be reduced. Alternatively, similar gains could be achieved by identifying periods of inactivity, and automatically preventing the recording and transmission of data at that time.

\section{REFERENCES}

[1] http://www.bbc.co.uk/news/health-33932930

[2] M. P. Rajasekaran, S. Radhakrishnan, and P. Subbaraj, "Elderly patient monitoring system using a wireless sensor network," Telemedicine and e-Health, vol.15, no.1, pp.73-79, Feb.2009.

[3] S.P.Lacour, I.Graz, D.Cotton, S.Bauer and S.Wagner, "Elastic components for prosthetic skin," 33rd Annual International Conference of the IEEE EMBS, Boston, Massachusetts, Aug.30 - Sep.3, 2011.

[4] A. V. Shirinov, and W. K. Schomburg, "Pressure sensor from a PVDF film," Sensors and Actuators A: Physical, vol.142, no.1, pp.4855,Mar.2008.

[5] X. Li, T. Reissman, F.Yu, and E.C.Kan, “A Low-Range Drift-Free Biocompatible Pressure Sensor Based on P(VDF-TrFE) Piezoelectric Thin Film,” MRS Proceedings. vol. 1222. Cambridge University Press, 2009.

[6] R.S Dahiya, P. Mittendorfer, M. Valle, G. Cheng, and V. J. Lumelsky, "Directions Toward Effective Utilization of Tactile Skin: A Review," IEEE Sensors Journal, vol.13, no.11, pp.4121-4138, Nov. 2013.

[7] S. Khan, W. Dang, L. Lorenzelli, R. Dahiya, Flexible Pressure Sensors based on Screen Printed P(VDF-TrFE) and P(VDFTrFE)/MWCNTs, IEEE Trans. Semiconductor Manufacturing, 2015 (in press), DOI 10.1109/TSM.2015.2468053

[8] N. Mathur, I. Glesk, and A.Buis, "Issues in Wearable Mobile Sensor Platform for Lower Limb Prosthetic Users," 17th International Conference on Transparent Optical Networks (ICTON), 5-7 July 2015, DOI: 10.1109/ICTON.2015.7193480

[9] N. Mathur, I. Glesk, and A.Buis, "Skin Temperature Prediction in Lower Limb Prostheses," IEEE Journal of Biomedical and Health informatics, in press, Nov. 2014. DOI: 10.1109/JBHI.2014.2368774 